\title{
Outcome after Acute Head Trauma Needing Neurosurgical Intervention in Patients with Oral Anticoagulants or Anti-Thrombotic Agents
}

\section{Auer $C^{*}$ and Wurm G}

Department of Neurosurgery, Landesnervenklinik Wagner Jauregg, Linz, Austria, Europe

\begin{abstract}
Introduction: The benefit-to-risk-ratio of anticoagulation has been discussed over years in the medical literature. Even in minor Traumatic Brain Injury (TBI), anticoagulation can cause an intracranial hemorrhage. However, there is few data in literature on outcome in TBI, anticoagulation and surgery. The aim was to determine the effect of anticoagulation on outcome and mortality after TBI.

Methods: We performed a retrospective review for patients after TBI needing neurosurgical intervention between January 2003 and September 2008. We compared patients with and without anticoagulation, regarding mortality and outcome. Outcome variables were Karnofsky perfomance score (KPS) and Glasgow outcome scale (GOS). Statistical testing was done by means of Fisher's exact test and a multiple logit model.

Results: 293 patients met inclusion criteria. 245 (83.6\%) were non anticoagulated patients, $48(16.4 \%)$ received oral anticoagulation (OAC) or antithrombotic agents (ATH). Mean age in all patients was 49.3 years.

Prothrombintime (PT), age, type of bleeding, neurological status on admission turned out as significant factors for mortality and outcome. Ranges of odds ratio for mortality went from 0.98 to 44.8 , for KPS and GOS from 0.06 to 1.3. Anticoagulation was not a significant predicting factor for worse outcome or mortality ( $p 0.886 ; 0.926 ; 0.934)$.

Conclusion: Age, neurological status on admission and compartment of bleeding are still the most significant prognostic factors for outcome after TBI. Low PT, higher age, comatose on admission, intracerebral hemorrhage, acute subdural hematomas and combined intracranial bleeding are factors predicting a worse outcome. Thus, the intensity of anticoagulation is an important factor, but not the use of these drugs itself.
\end{abstract}

Keywords: Traumatic brain injury; Surgery; Anticoagulation; Antithrombotic therapy; Outcome

\section{Introduction}

The use of Oral Anticoagulants (OAC) and Anti-thrombotic Medication (ATH) in patients with intermittent atrial fibrillation, other cardiac diseases, diabetes, peripheral vascular disease and other arteriopathic conditions has been proven to be of benefit, and their use is increasing. However, their use comes at risk of severe complications $[1,2]$. Warfarin for example reduces the risk of stroke by about twothirds from $5 \%$ to $2 \%$ per years, but is at a cost of 5-8 serious bleeding events per 1000 patient per year [1]. The benefit to risk ratio of anticoagulation and anti-thrombotic agents has been discussed over years in the medical literature.

Several studies have demonstrated that in case of traumatic brain injury (TBI) anticoagulation and anti-thrombotic therapy is associated with a seven- to ten-fold risk of intracranial hemorrhage [3,4]. Thus, the rate of mortality and morbidity far exceeds the rate in patients with similar head injuries who are not anticoagulated [3]. Additionally to high intensity of anticoagulation advanced age, as well as chronic alcohol abuse, liver disease and other bleeding diathesis, poorly controlled hypertension and poor clinical compliance have been linked to the early or late development of intracranial hemorrhage $[3,5]$. Even in the setting of minor head trauma, OAC-related intracranial hemorrhage has been noted [3,6-8]. Also Hart et al. [8] reported that even minor trauma is related to intracranial hemorrhage in OAC patients. The prolonged progression of symptoms probably reflects ongoing bleeding due to diminished capacity of clotting.

\section{Rationale of this study}

The prevalence of anticoagulation in head trauma has been examined in various studies. Most studies, however excluded the elderly (over 65 years), were not conducted consecutively, were only pilot studies [9], did not include surgical cases or mixed sporadic and traumatic cases [9]. Even though TBI patients with preinjury anticoagulation needing neurosurgical intervention are included in some reports in the published literature, no general statement on outcome and no recommendation for management can be derived from literature for this patient group yet.

Our impression was that anticoagulation therapy played a significant role in patients admitted to our department with head trauma, raising the question whether anticoagulation is influencing surgical strategies and outcome. There also appeared to be further morbidity after initial surgical treatment with an increased rate of rehemorrhage. This prompted a retrospective review of cases performed at our unit.

This retrospective analysis of a consecutive series of patients with TBI requiring neurosurgical interventions was undertaken to determine the effect of anticoagulation, if any, on outcome and on mortality, and to distinguish this effect from other influencing factors as age, type of bleeding etc.

*Corresponding author: Christian Auer, Department of Neurosurgery, Landesnervenklinik Wagner Jauregg Linz, Wagner Jauregg Weg 15, 4020 Linz, Austria, Europe,Tel. +437326921-25916; E-mail: christian.auer2@gespag.at

Received January 19, 2012; Accepted April 12, 2012; Published April 16, 2012

Citation: Auer C, Wurm G (2012) Outcome after Acute Head Trauma Needing Neurosurgical Intervention in Patients with Oral Anticoagulants or Anti-Thrombotic Agents. J Trauma Treat 1:132. doi:10.4172/2167-1222.1000132

Copyright: (c) 2012 Auer C, et al. This is an open-access article distributed under the terms of the Creative Commons Attribution License, which permits unrestricted use, distribution, and reproduction in any medium, provided the original author and source are credited. 


\section{Material \& Methods}

\section{Study population and setting}

We performed a retrospective single center study including all patients admitted to our department after an acute head trauma with intracranial bleeding, receiving an operation. Patient with head trauma without any operation, but under conservative treatment were excluded. All patients between January 2003 and September 2008 were included. There were 293 patients meeting inclusion criteria. Patients suffering from chronic subdural hemorrhage were excluded.

We specifically included documentation for the presence or absence of anticoagulation or anti-thrombotic therapy. The variables we examined were Prothrombintime (PT), patient age, sex, initial neurological examination, and radiological findings. Initial neurological examination was considered normal, abnormal, if there was documentation of focal weakness, alteration of consciousness, behavior or speech or cranial nerve abnormality and intubated and comatose. Radiological findings included skull fracture, epidural hematoma $(\mathrm{EDH})$, acute subdural hematoma $(\mathrm{aSDH})$, cerebral contusion (CC), intracerebral hematoma (ICH) and combined intracranial bleeding (cIB).

Follow-up time was between 6 months and 5 years. Follow up was determined by medical records and by physical examination, by phonecalls and questionnaire in the survivor group.

\section{Data analysis}

In an initial analysis we compared baseline characteristics of patients with and without anticoagulation. For categorical characteristics like sex, neurological status on admission, etc. Fisher's exact test was used, to compare the mean values of age and PT across the two groups a Student's t-test (unequal variances) was performed. Statistical significance was defined as a p-value $<0.05$.

To investigate the effect of anticoagulation on mortality, GOS and KPS a multiple logit model including all potential predictors and confounders was fitted. Significance of estimated effects was assessed using a Wald test. We report also estimated odds ratios which specify the effect of a covariate on the mortality odds. This is the factor by which mortality is multiplied for an increase of the covariate value by one, if the covariate is quantitative, and for a specific covariate category compared to the baseline category, if the covariate is categorical. 95\%confidence intervals (CI) are computed to assess the precision of the estimates. Covariate effects with a positive sign lead to an increase, effects with negative sign to a decrease of the odds of the highest outcome category versus lower outcome categories.

To analyse the effects of the covariates a separate analysis was carried out for each of these outcomes using the proportional odds model of McCullagh [10].

Additionally proportional odds models for each ordinal outcome variable were fitted using stepwise selection of covariates. Selected covariates were anticoagulation, PT, age, sex, status on admission and radiological findings and for mortality, GOS and KPS. Also in these parsimonious models the hypothesis of proportional odds was rejected.

\section{Results}

\section{Overall}

Over a period from January 2003 to September 2008 we treated a total of 293 patients meeting inclusion criteria. We examined a $100 \%$ of preinjury data. Follow up for the entire group was obtainable for 275 of 293 patients (93.9\%), although we performed consequent data mining.

Of all patients, $48(16.4 \%)$ received preinjury anticoagulation (group 1). The rest of the entire group, 245 patients (83.6\%) was non anticoagulated patients (group 2).

Regarding the use of anticoagulants, 25 patients (52.1\%) were using oral anticoagulation (OAC), and $23(47.9 \%)$ antithrombotic agents (ATH); details of different medication see Table 1.

Prothrombitime was included in the evaluation, with ranges from 8 to 122 . Mean PT in group 1 was 68.85 and 77.79 in group $2(\mathrm{p}=0.008)$. There was one patient with PT 8 and one with 9.

Mean age of the study population was 49.3 years, with ranges from 1 to 91 years. Mean age in group 1 was 73.53 (range $42-91$ ) years; mean age in group 2 was 44.67 (range $1-88)$ years $(\mathrm{p}<0.001)$. Ninety three $(31.7 \%)$ patients were female and $200(68.3 \%)$ were male. In group 2 , 69 of 245 patients were female (27.9\%), whereas a higher percentage, 24 of $48(50 \%)$ were female in group $1(\mathrm{p}=0.004)$. In both categories we saw significant differences between anti and non-anticoagulated patients.

Comparing anticoagulated and non-anticoagulated patients concerning the neurological status on admission, there were significant differences between them. In group 2 most patients were without any deficit, rather group 1, where most of them were comatose and intubated on admission (Table 2) $(\mathrm{p}=0.004)$.

A CT scan was obtained in all patients. Both groups showed differences in radiological findings. Anticoagulated patients had a trend to aSDH with a total of $70 \%$, whereas patients without anticoagulation did not show a tendency to any type of bleeding (Table 2$).(\mathrm{p}<0.001)$ Furthermore non-anticoagulated patients showed significant more skull fractures $(\mathrm{p}=0.002)$.

In all three analyses anticoagulation did not evidence as a significant factor, nor in mortality neither in GOS and KPS ( $\mathrm{p}=0.886$; $0.926 ; 0.934)$. Significant factors were PT, age, radiological finding and neurological status on admission.

A better outcome, connoted survival, high GOS and high KPS is

\begin{tabular}{|l|l|l|l|}
\hline Oral anticoagulation & Antithrombotic agents \\
\hline Phenprocoumon & $22(88 \%)$ & Acetylsalicylacid & $18(78.3 \%)$ \\
\hline Acenocumarol & $3(12 \%)$ & Clopidogrel & $1(4.3 \%)$ \\
\hline & & Combination & $4(17.4 \%)$ \\
\hline
\end{tabular}

Table 1: Medication.

\begin{tabular}{|l|l|l|l|}
\hline Age adjusted outcome & Mortality & GOS & KPS \\
\hline & odds ratio $(p)$ & \multicolumn{2}{l|}{} \\
\hline age & $1.55(<0.001)$ & $0.64(<0.001)$ & $0.64(<0.001)$ \\
\hline PT & $0.98(0.017)$ & $1.25(0.007)$ & $1.3(0.002)$ \\
\hline aSDH & $5.2(0.025)$ & $0.4(0.033)$ & $0.4(0.027)$ \\
\hline ICH & $44.8(<0.001)$ & $0.06(<0.001)$ & $0.07(<0.001)$ \\
\hline combined bleeding & $4.9(0.037)$ & $/$ & $0.39(0.033)$ \\
\hline comatose on admission & $12.57(0.005)$ & $0.2(<0.001)$ & $0.24(<0.001)$ \\
\hline anticoagulation & $\mathbf{( 0 . 8 8 6 )}$ & $\mathbf{( 0 . 9 2 6 )}$ & $\mathbf{( 0 . 9 3 4 )}$ \\
\hline
\end{tabular}

Abbreviations:

GOS - Glasgow Outcome Scale

KPS- Karnofsky Performance Score

PT- Prothrombin Time

aSDH- acute Subdural Hematoma

$\mathrm{ICH}$ - Intra Cerebral Hematoma 
conditional upon to higher PT, young age, without neurological findings on admission and type of bleeding. Odds ratios and significance see Table 2. Worse Outcome is most affected by combined bleeding, high age and bad neurological condition on admission. Furthermore low PT, aSDH, ICH are predicting worse outcome.

\section{Discussion}

Today, neurosurgeons are faced to a higher number of elderly patients, and the number of elderlies under OAC or ATH is increasing. The reason is that evidence shows a benefit preventing thromboembolic events [11]. Although there is this benefit, we know that the risk of spontaneous or traumatic bleeding is higher under anticoagulation $[11,12]$. The overall risk of hemorrhagic complications has been calculated by van de Meer et al. as 16.5 per 100 treatment per year, major bleeds counting for 2.7 per 100 treatment per year [13]. So the aim of our study was to see differences in mortality and outcome between anticoagulated and non-anticoagulated patients. Further, we wanted to determine important parameters for patient's outcome after TBI and surgical treatment.

Some previous studies had examined the effect of OAC or ATH on outcome after TBI. The results concerning the effect are quite different. Wojcik et al. and other authors did not show any differences in mortality $[4,14,15]$. On the other hand there is evidence showing differences in mortality and outcome [12,16,17]. All these studies combine conservative and surgical treatment and some merge acute trauma and chronically bleeding what leads to different outcome itself. In contrast this current study included only operated patients and only acute TBI. The results show that anticoagulation itself is not a predicting factor for mortality and morbidity. But, anticoagulation cannot be seen as black and white, the important thing is the intensity of anticoagulation.

It is becoming increasingly clear that the risks of bleeding events are proportional to the intensity of anticoagulation [12,18-20]. Levine et al. [20] observed in several studies a higher risk of fatal bleeding with high-intensity oral anticoagulant (OAC) therapy. Pieracci et al. [15] found in their series that the degree of anticoagulation, but not use of an anticoagulative drug itself, predicts adverse outcome after traumatic brain injury. Cerebral hemorrhage increases dramatically if the INR is $>4$, the absolute risk being $2 \%$ [18]. Hylek et al. [18] also found a doubling of risk with each 0.5 increase in PT. In this case the literature and our study got the same finding. The recent results find PT as one important factor. A patient with 1 point lower PT has the odds ratio 1.25 for a 1 step decreased GOS. Not seldom, patients are in a nontherapeutically range. Over anticoagulation is an obvious risk factor for hemorrhagic complications and should be avoided.

As our population ages and the majority of medical problems are diseases of the elderly (thrombembolic complications of atrial fibrillation, of deep venous thrombosis, vascular diseases, prosthetic cardiac valves, and so on) the use of anticoagulant and antithrombotic agents is expected to rise [6].

Age has consistently proven to be one of the most important factors of outcome from brain trauma $[6,21,22]$.

The overwhelming majority of patients receiving anticoagulation or anti-thrombotic therapy are the elderly (age over 65 years). Moreover, the response to anticoagulative agents is exaggerated with advancing age [23]. However, issues regarding indications and therapeutic ranges for the drugs remain disputed and are frequently not adjusted in relation to the aging process [3]. The risk/benefit equation of anticoagulation for the elderly seems more complex and differs from that for younger patients [3]. These things make age a confounding factor in Outcome. In the study of Cohen et al, mortality of brain trauma patients under anticoagulation in patients with GCS scores less than 8 was $91.5 \%$, with GSC scores of 13 to 15 mortality was $80.6 \%$. However, this seemingly excessive mortality must be questioned, considering that the average age in this series was 79 years and no calculation of this confounding factor has been performed [6]. This recent study had significant differences too, between anti and non-anticoagulated patient's age, and we stratified the groups in the statistic model. What we saw that age is the important factor and not anticoagulation. The older the patient, the worse was the chance to survive or to be in a healthy condition.

Clinical status on admission turned out as one of the most important factors for outcome in our study. This confirms the findings of Cohen et al. who found a mortality rate of $87.8 \%$ for patients with GCS scores less than 8 [6].

High mortality of aSDH and ICH is described in many studies already from the early 60 's to the present $[24,25]$. Not only mortality, also outcome is influenced by the type of bleeding. Our results showed, that combined hemorrhage, aSDH and ICH cause a higher mortality and a worse outcome, especially ICH with an odds ratio of nearly 45 compared to epidural hematomas concerning mortality.

A significant difference in skull fractures between anticoagulated and non anticoagulated patients seems to be a sign for different type of injuries. Intracranial hemorrhage appears even in minor trauma in anticoagulated and especially over anticoagulated patients $[6,8]$. Our study has a lack of type and severtity of trauma, but the differences in skull fractures seem to show differences in severity of trauma, compatible to literature.

\section{Limitations and future questions}

There are several limitations to this study that should be considered. First, our data were retrospectively abstracted from our hospital records, with no mechanism to validate the accuracy of measurements. Prehospital and admission GCS values as well as the reason for preinjury anticoagulation could not be found in a considerable part of patients, thus were not included in our calculations. Second, OAC and ATH were put together as one group, thus it is different medication. Statistical analysis in the subgroups of medication could not be performed due to small patient series in the subgroups. Finally, reversal of anticoagulation was not performed in a regular manner with strict dosage regime, even though clotting factor concentrates have been used in the majority of cases, further not in every patient with ATH medication a platelet function test was performed to proof, if they were responder.

\section{Conclusion}

Given the fact that TBI is the fifth leading cause of death in the age group where the use of anticoagulation is gaining popularity, it is highly likely that neurosurgeons and trauma surgeons will be confronted with the clinical scenario of traumatic brain injury in an anticoagulated patient on a regular basis. Therefore, outcome data for this patient group are highly warranted. Our results correlate strongly with previous suggestions that the prognosis of intracranial hemorrhage is predominantly influenced by the patient's age, the clinical status, the compartment of bleeding and the degree of anticoagulation at the time of presentation. This underscores the importance of ongoing investigations as to the early reversal of anticoagulation as well as to optimal values of INR and PT in these patients, but most important is 
Citation: Auer C, Wurm G (2012) Outcome after Acute Head Trauma Needing Neurosurgical Intervention in Patients with Oral Anticoagulants or AntiThrombotic Agents. J Trauma Treat 1:131. doi:10.4172/2167-1222.1000132

that anticoagulation itself is not a predicting factor for outcome after TBI.

\section{References}

1. Rust T, Kiemer N, Erasmus A (2006) Chronic subdural haematomas and anticoagulation or anti-thrombotic therapy. J Clin Neurosci 13: 823-827.

2. Levine MN, Anderson DR (1990) Side-effects of antithrombotic therapy. Baillieres Clin Haematol 3: 815-829.

3. Karni A, Holtzman R, Bass T, Zorman G, Carter L, et al. (2001) Traumatic head injury in the anticoagulated elderly patient: a lethal combination. Am Surg 67: 1098-1100.

4. Kennedy DM, Cipolle MD, Pasquale MD, Wasser T (2000) Impact of preinjury warfarin use in elderly trauma patients. J Trauma 48: 451-453.

5. Pentland B, Jones PA, Roy CW, Miller JD (1986) Head injury in the elderly. Age Ageing 15: 193-202.

6. Cohen DB, Rinker C, Wilberger JE (2006) Traumatic brain injury in anticoagulated patients. J Trauma 60: 553-557.

7. Volans AP (1998) The risks of minor head injury in the warfarinised patient. Accid Emerg Med 15: 159-161.

8. Hart RG, Boop BS, Anderson DC (1995) Oral anticoagulants and intracrania hemorrhage. Facts and hypotheses. Stroke 26: 1471-1477.

9. Cartmill M, Dolan G, Byrne JL, Byrne PO (2000) Prothrombin complex concentrate for oral anticoagulant reversal in neurosurgical emergencies. $\mathrm{Br} \mathrm{J}$ Neurosurg 14: 458-461.

10. http://people.csail.mit.edu/jrennie/papers/other/mccullagh-ordinal-80.pdf

11. Hirsh J, Dalen J, Anderson DR, Poller L, Bussey H, et al. (2001) Ora anticoagulants: mechanism of action, clinical effectiveness, and optimal therapeutic range. Chest 119: 8S-21S

12. Mathiesen T, Benediktsdottir K, Johnsson H, Lindqvist M, von Holst H (1995) Intracranial traumatic and non-traumatic haemorrhagic complications of warfarin treatment. Acta Neurol Scand 91: 208-214.

13. van der Meer FJ, Rosendaal FR, Vandenbroucke JP, Briët E (1993) Bleeding complications in oral anticoagulant therapy. An analysis of risk factors. Arch Intern Med 153: 1557-1562.

14. Wojcik R, Cipolle MD, Seislove E, Wasser TE, Pasquale MD (2001) Preinjury warfarin does not impact outcome in trauma patients. J Trauma 51: 1147-1151.

15. Pieracci FM, Eachempati SR, Shou J, Hydo LJ, Barie PS (2007) Degree of anticoagulation, but not warfarin use itself, predicts adverse outcomes after traumatic brain injury in elderly trauma patients. J Trauma 63: 525-530.

16. Franko J, Kish KJ, O'Connell BG, Subramanian S, Yuschak JV (2006) Advanced age and preinjury warfarin anticoagulation increase the risk of mortality after head trauma. J Trauma 61: 107-110.

17. Mina AA, Knipfer JF, Park DY, Bair HA, Howells GA, et al. (2002) Intracrania complications of preinjury anticoagulation in trauma patients with head injury. J Trauma 53: 668-672

18. Hylek EM, Singer DE (1994) Risk factors for intracranial hemorrhage in outpatients taking warfarin. Ann Intern Med 120: 897-902.

19. Kase CS, Robinson RK, Stein RW, DeWitt LD, Hier DB, et al. (1985) Anticoagulant-related intracerebral hemorrhage. Neurology 35: 943-948.

20. Levine MN, Hirsh J, Landefeld S, Raskob G (1992) Hemorrhagic complications of anticoagulant treatment. Chest 102: 352S-363S.

21. Mosenthal AC, Livingston DH, Lavery RF, Knudson MM, Lee S, et al. (2004) The effect of age on functional outcome in mild traumatic brain injury: 6-month report of a prospective multicenter trial. J Trauma 56: 1042-1048.

22. Nagurney JT, Borczuk P, Thomas SH (1998) Elder patients with closed head trauma: a comparison with nonelder patients. Acad Emerg Med 5: 678-684.

23. Gurwitz JH, Avorn J, Ross-Degnan D, Choodnovskiy I, Ansell J (1992) Aging and the anticoagulant response to warfarin therapy. Ann Intern Med 116: 901 904

24. McKissock W, Richardson A, Bloo WH (1960) Subdural hematoma: a review of 389 cases. Lancet 1: 1365-1369.

25. Taussky P, Widmer HR, Takala J, Fandino J (2008) Outcome after acute traumatic subdural and epidural haematoma in Switzerland: a single-centre experience. Swiss Med Wkly 138: 281-285. 